\title{
Telemetry System Utilization for Stress Monitoring of Pilots During Training
}

\section{Luboš Socha ${ }^{1}$, Lenka Hanáková2*, Vladimír Socha ${ }^{2}$, Andrej Lališ², Róbert Rozenberg ${ }^{3}$, Karel Hána ${ }^{4}$}

\footnotetext{
${ }^{1}$ Department of Air Transport Management, Faculty of Aeronautics, Technical University of Košice, Košice, Slovak Republic

${ }^{2}$ Department of Air Transport, Faculty of Transportation Sciences, Czech Technical University in Prague, Prague,

Czech Republic

${ }^{3}$ Department of Flight Training, Faculty of Aeronautics, Technical University of Košice, Košice, Slovak Republic

${ }^{4}$ University Centre for Energy Efficient Buildings, Czech Technical University in Prague, Bustehrad, Czech Republic

*Corresponding author: Czech Technical University in Prague, Faculty of Transportation Sciences, Department of Air

Transport, Horská 3, 12803 Prague, Czech Republic, Email: hanakle1@fd.cvut.cz
}

\begin{abstract}
Air transport development brings an increased focus on the safety of piloting. The safety conditions can be assessed by mental workload. Psychic discomfort or excessive stress on pilots can negatively influence the course of flights. Therefore it appears convenient to monitor such parameters, which represent the mental wellbeing, or discomfort of a pilot. Since physiological measurements can provide a good information about mental workload or stress, this work primarily focuses on the observation of the change in heart rate, as it is an indicator of stress during the training of pilots, using the designed modular telemetry system. Another aim of this study is to evaluate the influence of a change in the avionic data visualization. This can have an unfavorable effect on the piloting of an airplane. This work, based on the evaluation of heart rate shows, that the switch from analog visualization to glass cockpit creates increased levels of stress in pilots, which was proved for all examined subjects except one. Significant level of correlation in the heart beat rate change in subjects in the course of training was also discovered.
\end{abstract}

Keywords

Aviation - Heart rate - Mental workload - Mental stress - Telemetry system

\section{Introduction}

During piloting an airplane it is imporant to simultaneously process numerous information from different sources. This is due to the increasing intellectualization and informatization of air transport and the development of the cockpit information interface [1]. Today the main task of the pilot is not manually control the aircraft, but check all flight information and respond to them [2]. Modern aircraft cockipt is a so-called glass cockpit, featuring various digital displays, which should be highly intuitive. But same as for analog data visualization, a complex system still lags behind the scenes. As a result, 
there is an increasing demand on pilots to monitor all displays in modern aircraft [3]. Consequently, pilots mental stress increases and limitations in monitoring of environment emerge which lead to decreased situational awareness [2, 4].

Psychological state can be evaluated subjectively and objectively. Subjective evaluation is comprised of questionnaires which, however, cannot be filled in-flight and so it is impossible to gather information about mental state of monitored person [5, 6]. By contrast, objective evaluation is based on biosignal measurement which enables online mental state monitoring. Among the most observed values in the evaluation of mental pressure, there are various physiological parameters. The most frequently observed value is the heart rate, and other bodily functions, which directly or indirectly reflex the activity of the autonomic nervous system [4, 5, 7, 8]. Generally, in stressful situations, the autonomic nervous system activates its sympathetic sub-system, increasing heart and respiratory rate and blood pressure, as well as blood flow in skeletal muscles, and decreasing blood flow in smooth muscles. This also elevates sensory perception (dilated pupils), activates energy reserve, etc. On the other hand, parasympathetic nervous system is active outside stressful situations, and its effects are basically the opposite of those of sympathetic nervous system. It slows down the heart and respiratory rate, increases the blood flow in smooth muscles, etc. [9]. Thus, using indicators such as heart rate, it is possible to evaluate whether or not the observed subject is under stress. A typical feature of flying modern aircraft is the constant increase in demands on mainly psychic activity of the pilots [5, 7], which points out to the necessity of monitoring psychophysiological parameters. The field of collecting and processing physiological data of pilots during the flight is a widely discussed subject. It was proven, that the measured physiological quantities are influenced by the change in the difficulty of flight tasks both in real flights, and flights on a simulator [10, 11]. It was also discovered, that the level of pilot's mental stress depends on the level of his training [12]. Psychic discomfort of the pilot, high level of stress and lack of experience can influence the course of a flight negatively. Human factor is responsible for $70-80 \%$ of aviation accidents, out of which $50 \%$ is caused directly by pilots [13]. Since human factor faultiness in the pilot's decisionmaking contributes significantly to the number of accidents [14], it is apparent, that monitoring parameters describing mental, or physical stress of the pilot could contribute to the improvement of flight safety. The designing the systems for dynamic monitoring of psychophysiological parameters can aid to recognize the pilot fatigue borderlines, decrease in concentration, or current physical state of the pilot [4].

The main objective of this study is to describe the applicability of the FlexiGuard telemetry system, developed at the Joint Department of Biomedical Engineering CTU and Charles University in Prague, for the purposes of monitoring the pilots' stress, on the basis of the examination of the influence of the change in avionic data visualization on pilots in training. The null hypothesis is that the measured heart rate, as the stress indicator, will decrease in participants during the simulator training, increase during real flights and change significantly with the change in avionic data visualization from analog to digital. The main cause for the assumed change in the pilot's condition within the change in the avionic data visualization is that the pilot's aptitude is limited to the type of an aircraft, but cockpit ergonomics, as for the visualization of the avionic data, is not taken into account at all. The issue of the type of visualization of navigation and engine data on the cockpit dashboard of a particular type of aircraft is not resolved. Such change can enable a pilot, who has experience only with flights using analog visualization for the given type of aircraft, to accomplish the flight using glasscockpit visualization, without any retraining or theoretical training on the same aircraft. Such situations occur mainly in the field of general aviation.

\section{Materials and methods}

\subsection{Participants and measuring procedure}

Eight students of the Faculty of Aeronautics of the Technical University of Kosice participated in this study. All subject passed theoretical and psychological tests. The goal of the testing was to assure participants with good orientation during stress situations. Besides the testing, all participants had to comply with medical requirements (Commission Regulation (EU) No 1178/2011- Annex IV) and simultaneously couldn't be holders of pilot license (PPL) what assured maximum uniformity possible of the monitored subjects. The participants first completed a theoretical training, followed by 11 hour training on a simulator TRD40 with an analog display of avionic data. The heart rate measurement took place in the second (S2M) and eleventh lesson of the training (S11M). After the initial training on the simulator, the training continued on Diamond DA40 aircraft with analog display of avionic data, where measurements took place in the first (A12M) and the third flight lesson AL17M). Pause between flights was designed for the training on the simulator. During every following lesson, the way of the avionic data visualization was changed from analog to digital (glass cockpit), where the measurements S18M on the flight simulator, and A19M on Diamond DA 40 took place. In the course of the training, besides the takeoff and landing, three series of maneuvers were exercised, including flying at constant altitude, $360^{\circ}$ turn, and $180^{\circ}$ climbing and descending turn at the vertical speed of $500 \mathrm{ft} / \mathrm{min}$. The congruence of all training lessons was accomplished by a precise designation of the course of the flight based on the aforementioned maneuvers.

\subsection{Measuring equipment}

The measurements were realized using the FlexiGuard system, developed at the Joint department of Biomedical Engineering CTU and Charles University in Prague. The system consists of three layers. The first layer contains sensors. Sensing signal in the form of beats-per-minute and RR intervals was sent wirelessly to the second layer ensuring the data acquisition 
with the sampling frequency of $5 \mathrm{~Hz}$. The central unit, which the second layer consists of, processes and partly evaluates the data received from the sensors. These modified data were send to the third layer by the central unit. The last layer of the system consists of the visualization unit and a respective application for the representation of the measured data. The designed software allows inserting time marks during the measurement and this way, the beginning and end of flight was marked just like all performed maneuvers. The system is primarily designed and intended for members of the integrated rescue system, but due to its modularity, it is possible to adapt it for the monitoring of pilots. For the measurement of heart rate, a Garmin commercial wireless sensor (Garmin HRM 3 Premium Soft Chest strap) was used within the FlexiGuard system, using ANT+ protocol to communicate with the control unit. Belt for scanning heart beat rate was located under precessus xiphoideus, Fig. 1. Sensing signal in the form of beats-per-minute (bpm) and RR intervals was sent to the second layer using ANT+ protocol to communicate with the control unit. The data was collected with sampling frequency of $5 \mathrm{~Hz}$.

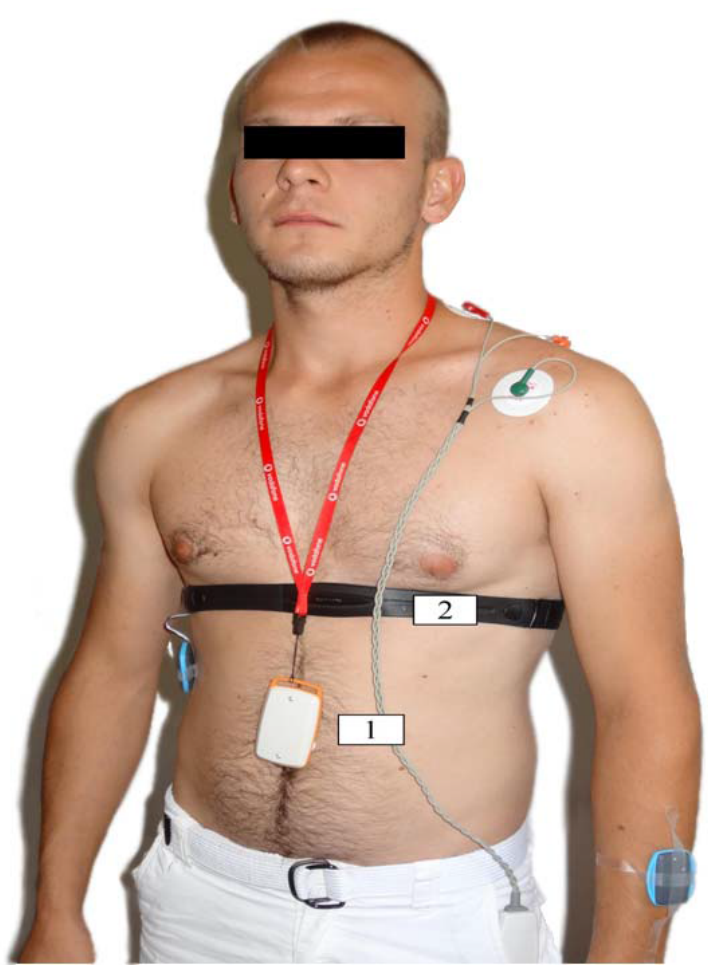

Figure 1. Placing of the sensors on the subject; central unit (1), heart rate beat sensor - Garmin belt (2).

\subsection{Data processing and statistical analysis}

Data representing bpm were collected. Average values from the data measured in individual flights (training phases) were calculated for the purpose of obtaining mean values of heart rate, based on which the course of the training process was evaluated. Based on Jarque-Bera testing [15] it was deter- mined, that mean values of heart rate are not normally distributed. Therefore, Spearman correlation coefficient [16] was used in the comparison of heart beat rates.

For the purpose of signal processing and statistical analysis, a custom-designed MatLab (MatLab R2013a, Mathworks, Inc., Natick, MA, USA) programs were used.

\section{Results}

Mean values were calculated from the measured heart beat rate (Tab. 1). A decrease of heart beat rate was observed in five subjects in the measurement S2M compared with S11M. The most significant deterioration was measured in subject 6 with the increase of $5.08 \mathrm{bpm}$. A rapid increase of heart beat rate (at an average of $17.14 \mathrm{bpm}$ (SD $7.91 \mathrm{bpm}$ )) was found in all subjects, during the first realization of training on the Diamond DA40 aircraft in real traffic. An increase was recorded in one subject in A17M measurement, at an average of $27.21 \mathrm{bpm}$. After the change to the digital visualization of avionic data within the simulator measurement, the heart beat rate increased in half of the subjects by $8.59 \mathrm{bpm}$ (SD $6.26 \mathrm{bpm}$ ) and decreased in the rest of subjects by $4.92 \mathrm{bpm}$ (SD $1.51 \mathrm{bpm}$ ) when comparing measurements of S11M and S18M.

Except for subject 4, all subjects show an increase of heart beat rate in the last measurement when compared to A17M. By comparing the last measurement, during which the participants piloted the aircraft with the aid of the digital visualization of avionic data, to the case with analog visualization, the results show an increase of heart beat rate of $14.76 \mathrm{bpm}$ (SD 6.63 bpm).

For statistical comparison of heart beat rates, the Spearman correlation coefficient was used. Table 2 demonstrates the results representing correlations of heart beat rates for all subjects. Correlation coefficients were calculated for the purpose of discovering the incidence of graphs, and therefore the compliance of the heart beat rate distribution in the course of training. By testing the hypothesis that the correlation coefficient is significantly different from 0 on the significance level $p=0.15$ (in our case it means the correlation coefficient is higher than 70) it was found, that compliance of graphs is $46.42 \%$. This means, that out of 28 combinations, 13 comply (are identical to each other). The course of the heart beat distribution complies the least in subject 4 .

\section{Discussion and conclusion}

For pilot observation purposes, modified FlexiGuard system was used to evaluate pilots' stress levels in this study. A part of the system's output is a direct feed of measurement progress heart beat rate in real time in this case. Though quite simple and utilizing commercial devices, the system is relatively well applicable for physiological parameters observation. Apart from being able to supply an output device (tablet, PC, etc.) with acquired data, the system also provides a central storage 
Table 1. Mean values of the heart beat rate (in bpm) of subjects in individual measurements.

\begin{tabular}{ccccccc}
\hline & S2M & S11M & A12M & A17M & S18M & A19M \\
\hline 1 & 92.14 & 84.01 & 101.60 & 90.54 & 93.77 & 93.89 \\
\hline 2 & 82.21 & 72.52 & 90.01 & 80.21 & 68.26 & 87.84 \\
\hline 3 & 59.08 & 62.28 & 75.58 & 69.90 & 55.70 & 82.47 \\
\hline 4 & 99.29 & 81.14 & 84.26 & 111.47 & 83.44 & 107.76 \\
\hline 5 & 93.45 & 85.55 & 94.88 & 94.06 & 89.52 & 115.90 \\
\hline 6 & 82.69 & 87.77 & 110.56 & 97.96 & 84.98 & 111.19 \\
\hline 7 & 79.58 & 81.62 & 106.16 & 91.27 & 75.54 & 116.89 \\
\hline 8 & 81.15 & 71.47 & 100.47 & 87.95 & 89.79 & 91.74 \\
\hline
\end{tabular}

Table 2. Spearman correlation coefficient for the comparison of the graphs.

\begin{tabular}{ccccccccc}
\hline Subj. & 1 & 2 & 3 & 4 & 5 & 6 & 7 & 8 \\
\hline 1 & - & 0.66 & 0.43 & 0.14 & 0.71 & 0.49 & 0.43 & 0.94 \\
\hline 2 & 0.66 & - & 0.77 & 0.43 & 0.83 & 0.60 & 0.77 & 0.60 \\
\hline 3 & 0.43 & 0.77 & - & 0.49 & 0.83 & 0.94 & 1.00 & 0.54 \\
\hline 4 & 0.14 & 0.43 & 0.49 & - & 0.71 & 0.37 & 0.49 & 0.26 \\
\hline 5 & 0.71 & 0.83 & 0.83 & 0.71 & - & 0.77 & 0.83 & 0.77 \\
\hline 6 & 0.49 & 0.60 & 0.94 & 0.37 & 0.77 & - & 0.94 & 0.66 \\
\hline 7 & 0.43 & 0.77 & 1.00 & 0.49 & 0.83 & 0.94 & - & 0.54 \\
\hline 8 & 0.94 & 0.60 & 0.54 & 0.26 & 0.77 & 0.66 & 0.54 & - \\
\hline
\end{tabular}

capacity for further evaluation. Obtained data were used in stress levels' evaluation procedure.

Based on observing pilots in training it was found that the course of the training influences their mental wellbeing. Even though our sample consisted of relatively small number of subjects, it is apparent that, for example, during the switch from the simulator to the aircraft, heart beat rate increased in all subjects, which can be defined as and indicator of stress [17, $18]$.

The hypothesis about the heart beat rate during the course of the training was proven in five subjects. In any case, the trend did not change rapidly. It was further found, that in inexperienced pilots, comprising the examined sample, the switch from analog visualization of avionic data to digital was problematic. We assume, that this was caused by a lack of preparation for a different kind of visualization, since the schedule for the training plan contained only one hour of preparation on glass cockpit.

Some limitations of this work might be measuring "only" the heart beat rate, or rather small size of the sample. In any case, this work points out to the necessity of the further research of the pilots' stress levels. In future studies, it would be interesting to observe and evaluate more parameters, such as myopotentials, movement activity, respiratory rate, etc, using modular systems such as FlexiGuard. These systems can further serve for dynamic examination of the pilot's condition and provide feedback to instructors, or aircraft traffic control, or find use in the clinics of aviation health service. Aforementioned findings and proposals can positively influence the improvement of aviation safety.

\section{Acknowledgments}

This research was funded by Ministry of Education, Science, Research and Sport of the Slovak Republic within execution of the project No. ITMS 26220220161 (Research of pilots training methods using flight simulator) co-financed by EU funds. This work was also supported by the Ministry of Education, Youth and Sports of Czech Republic within National Sustainability Programme I, project No. LO160. The authors would like to thank Andrej Madoran, B.A., for translation and language check of this work.

\section{References}

[1] H. Zhang, X. Wanyan, D. Zhuang, and X. Wu. Study on comprehensive evaluation method of pilot mental workload. In 2014 Sixth International Conference on Intelligent Human-Machine Systems and Cybernetics. Institute of Electrical \& Electronics Engineers (IEEE), aug 2014. doi: 10.1109/ihmsc.2014.35. URL http: //dx.doi.org/10.1109/ihmsc.2014.35.

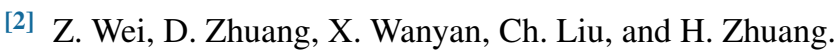
A model for discrimination and prediction of mental workload of aircraft cockpit display interface. Chinese Journal of Aeronautics, 27(5):1070-1077, oct 2014. doi: 10.1016/j.cja.2014.09.002. URL http://dx.doi. org/10.1016/j.cja.2014.09.002.

${ }^{[3]}$ G. Borghini, L. Astolfi, G. Vecchiato, Do. Mattia, and Fa. Babiloni. Measuring neurophysiological signals in aircraft pilots and car drivers for the assessment of mental workload, fatigue and drowsiness. Neuroscience \& 
Biobehavioral Reviews, 44:58-75, jul 2014. doi: 10. 1016/j.neubiorev.2012.10.003. URL http: / / dx . doi . org/10.1016/j.neubiorev.2012.10.003.

[4] A. Haarmann, W. Boucsein, and F. Schaefer. Combining electrodermal responses and cardiovascular measures for probing adaptive automation during simulated flight. Applied Ergonomics, 40(6):1026-1040, nov 2009. doi: 10.1016/j.apergo.2009.04.011. URL http: / / dx . doi . org/10.1016/j.apergo.2009.04.011.

[5] J.A. Veltman and A.W.K. Gaillard. Physiological indices of workload in a simulated flight task. Biological Psychology, 42(3):323-342, feb 1996. doi: 10. 1016/0301-0511(95)05165-1. URL http: / / dx . doi. org/10.1016/0301-0511(95)05165-1.

[6] M. Causse, J.-M. Sénard, J.F. Démonet, and J. Pastor. Monitoring cognitive and emotional processes through pupil and cardiac response during dynamic versus logical task. Applied Psychophysiology and Biofeedback, 35(2):115-123, oct 2009. doi: 10.1007/ s10484-009-9115-0. URL http://dx.doi.org/ $10.1007 / \mathrm{s} 10484-009-9115-0$.

[7] B. Johannes, V. Salnitski, H. Soll, M. Rauch, and H.-J. Hoermann. De-individualized psychophysiological strain assessment during a flight simulation test-validation of a space methodology. Acta Astronautica, 63(710):791-799, oct 2008. doi: 10.1016/j.actaastro.2008. 03.017. URL http://dx.doi.org/10.1016/j. actaastro.2008.03.017.

[8] P. Lehrer, M. Karavidas, S.-E. Lu, E. Vaschillo, B. Vaschillo, and A. Cheng. Cardiac data increase association between self-report and both expert ratings of task load and task performance in flight simulator tasks: An exploratory study. International Journal of Psychophysiology, 76(2):80-87, may 2010. doi: 10.1016/ j.ijpsycho.2010.02.006. URL http: / / dx. doi .org/ $10.1016 / j . i j p s y c h o .2010 .02 .006$.

[9] C. Schubert, M. Lambertz, R.A. Nelesen, W. Bardwell, J.-B. Choi, and J.E. Dimsdale. Effects of stress on heart rate complexity - a comparison between shortterm and chronic stress. Biological Psychology, 80(3): 325-332, mar 2009. doi: 10.1016/j.biopsycho.2008. 11.005. URL http://dx.doi.org/10.1016/j. biopsycho.2008.11.005.

[10] D.W. Watson. Physiological correlates of heart rate variability (HRV) and the subjective assessment of workload and fatigue in-flight crew: a practical study. In People in Control. Human Factors in Control Room Design. Institution of Engineering and Technology (IET), 2001. doi: 10.1049/cp:20010453. URL http://dx.doi.org/ $10.1049 / \mathrm{cp}: 20010453$.
[11] Ch. Papadelis, Ch. Kourtidou-Papadeli, P. Bamidis, and M. Albani. Effects of imagery training on cognitive performance and use of physiological measures as an assessment tool of mental effort. Brain and Cognition, 64 (1):74-85, jun 2007. doi: 10.1016/j.bandc.2007.01.001. URL http://dx.doi.org/10.1016/j.bandc. 2007.01 .001 .

[12] M.A. Bonner and G.F. Wilson. Heart rate measures of flight test and evaluation. The International Journal of Aviation Psychology, 12(1):63-77, jan 2002. doi: 10. 1207/s15327108ijap1201_6. URL http:// dx.doi . org/10.1207/s15327108ijap1201_6.

[13] National Transportation Safety Board. Annual review of aircraft accident data, U.S. general aviation, calendar year 2005, 2005. URL http: / / www . dviaviation. com/files/38801058.pdf. [Online].

[14] R. Rozenberg and S. Szabo. Criteria of selecting pilots for aerobatic teams. In Aeronautika 2013. Technical University of Kosice, 2013.

[15] C.M. Jarque and A.K. Bera. A test for normality of observations and regression residuals. International Statistical Review / Revue Internationale de Statistique, 55(2):163, aug 1987. doi: 10.2307/1403192. URL http://dx.doi.org/10.2307/1403192.

[16] L. Myers and M.J. Sirois. Spearman correlation coefficients, differences between. Wiley StatsRef: Statistics Reference Online, 2006. doi: 10.1002/9781118445112. stat02802. URL http://dx.doi.org/10.1002/ 9781118445112. stat02802.

[17] J.B. Bricker. Development and evaluation of the air travel stress scale. Journal of Counseling Psychology, 52(4):615, 2005. doi: 10.1037/0022-0167. 52.4.615. URL http://dx.doi.org/10.1037/ 0022-0167.52.4.615.

[18] R. Orsila, M. Virtanen, T. Luukkaala, M. Tarvainen, P. Karjalainen, J. Viik, M. Savinainen, and C.-H. Nygård. Perceived mental stress and reactions in heart rate variability - a pilot study among employees of an electronics company. International Journal of Occupational Safety and Ergonomics, 14(3):275-283, jan 2008. doi: 10.1080/ 10803548.2008.11076767. URL http://dx.doi. org/10.1080/10803548.2008.11076767. 